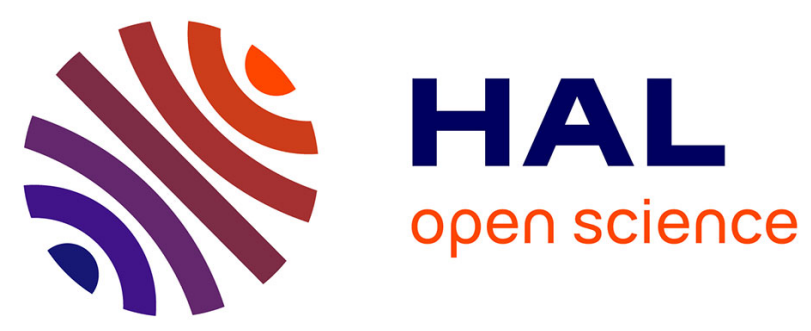

\title{
Measurements of ultrasound velocity and attenuation in numerical anisotropic porous media compared to Biot's and multiple scattering models.
}

\author{
Fabien Mézière, Marie Muller, Emmanuel Bossy, Arnaud Derode
}

\section{- To cite this version:}

Fabien Mézière, Marie Muller, Emmanuel Bossy, Arnaud Derode. Measurements of ultrasound velocity and attenuation in numerical anisotropic porous media compared to Biot's and multiple scattering models.. Ultrasonics, 2014, 54 (5), pp.1146-54. 10.1016/j.ultras.2013.09.013 . inserm-00877374

\section{HAL Id: inserm-00877374 https://www.hal.inserm.fr/inserm-00877374}

Submitted on 28 Oct 2013

HAL is a multi-disciplinary open access archive for the deposit and dissemination of scientific research documents, whether they are published or not. The documents may come from teaching and research institutions in France or abroad, or from public or private research centers.
L'archive ouverte pluridisciplinaire HAL, est destinée au dépôt et à la diffusion de documents scientifiques de niveau recherche, publiés ou non, émanant des établissements d'enseignement et de recherche français ou étrangers, des laboratoires publics ou privés. 


\title{
Measurements of ultrasound velocity and attenuation in numerical anisotropic porous media compared to Biot's and multiple scattering models
}

\author{
Fabien Mézière, Marie Muller, Emmanuel Bossy, Arnaud Derode \\ Institut Langevin, ESPCI ParisTech, CNRS UMR7587, INSERM U979, Université Paris Diderot - Paris 7, 1 rue Jussieu, 75005 Paris, France
}

\begin{abstract}
This article quantitatively investigates ultrasound propagation in numerical anisotropic porous media with finite-difference simulations in 3D. The propagation media consist of clusters of ellipsoidal scatterers randomly distributed in water, mimicking the anisotropic structure of cancellous bone. Velocities and attenuation coefficients of the ensemble-averaged transmitted wave (also known as the coherent wave) are measured in various configurations. As in real cancellous bone, one or two longitudinal modes emerge, depending on the micro-structure. The results are confronted with two standard theoretical approaches: Biot's theory, usually invoked in porous media, and the Independent Scattering Approximation (ISA), a classical first-order approach of multiple scattering theory. On the one hand, when only one longitudinal wave is observed, it is found that at porosities higher than $90 \%$ the ISA successfully predicts the attenuation coefficient (unlike Biot's theory), as well as the existence of negative dispersion. On the other hand, the ISA is not well suited to study two-wave propagation, unlike Biot's model, at least as far as wave speeds are concerned. No free fitting parameters were used for the application of Biot's theory. Finally we investigate the phase-shift between waves in the fluid and the solid structure, and compare them to Biot's predictions of in-phase and out-of-phase motions.
\end{abstract}

Keywords: Cancellous bone, Fast and slow waves, Porous media, Multiple scattering, FDTD simulations, Biot's theory

\section{Introduction} derstood.
Cancellous bone is a random and porous material with struc- ${ }^{31}$ tural anisotropy. Ultrasound transmission experiments revealed ${ }^{32}$ that in some cases two compressional waves propagating at ${ }^{33}$ different velocities were observed, as reported both in vitro ${ }^{34}$ $[1,2,3]$ and in vivo [4]. Yet this phenomenon is not fully un- ${ }^{35}$

From a theoretical point of view, there are several ways to ${ }^{37}$ account for wave propagation in media as complex as cancel- ${ }^{38}$ lous bone. A very simple approach, which will be referred to ${ }^{39}$ as Wood's model [5], consists in considering bone as a homo- ${ }^{40}$ geneous medium in which sound speed is determined from the ${ }^{41}$ averaged mass densities and compliances of the solid and fluid ${ }^{42}$ phases, weighted by their respective volumetric fractions. $A^{43}$ more elaborate theoretical description is given by Biot $[6,7]$. $^{44}$ Though it is out of the scope of this paper to give a full descrip- ${ }^{45}$ tion of Biot's model, let us recall that it is an homogeneiza- ${ }^{46}$ tion theory, like Wood's model. Biot's theory is a reference ${ }^{47}$ model for wave propagation in porous media, particularly be- ${ }^{48}$ cause it was shown to predict successfully the velocities of two ${ }^{49}$ longitudinal waves in various porous media, from sintered glass 50 spheres [8] to cortical [9] and cancellous bone [2, 10]. However, ${ }^{51}$ the validity of Biot's model is limited to low frequencies. Biot ${ }^{52}$ [7] himself wrote: "There remains however an upper bound for ${ }^{53}$ the frequency, namely, that at which the wavelength becomes ${ }^{54}$ of the order of the pore size. Such a case must, of course, ${ }^{55}$ be treated by a different method." Considering typical dimen- ${ }^{56}$ sions at stake, in the $\mathrm{MHz}$ domain, the ultrasonic wavelengths ${ }^{57}$ are of the same order of magnitude as the size of the trabeculae [11, chap.1]. An immediate consequence is that single and even multiple scattering must be taken into account [12]. Scattering induce loss, which is not predicted by Biot's theory: as long as the fluid phase is free of absorption, Biot's fast and slow waves do not show any attenuation. Moreover, scattering does not only affect the wave amplitude, but also its velocity, though to a lesser degree. An alternative approach to Biot's would be to adopt a multiple scattering formalism for wave propagation in cancellous bone. A given sample is treated as one realization of a random process, whose typical physical parameters (density $\rho$, elastic moduli $C_{i j k l}$ ) randomly depend on position $\vec{r}$ within the medium. Assuming gaussian statistics, the microstructure would be characterized by second-order moments i.e., correlation functions such as $\left\langle\rho(\vec{r}) \rho\left(\overrightarrow{r^{\prime}}\right)\right\rangle,\left\langle C_{i j k l}(\vec{r}) C_{\text {mnop }}\left(\overrightarrow{r^{\prime}}\right)\right\rangle$ etc. In the random multiple scattering approach, the wave field $s(\vec{r}, t)$ resulting from the emission of a pulse through a slice of bone is considered as a random variable. The basic quantities of interest are its statistical average $\langle s(\vec{r}, t)\rangle$ (also referred to in the literature as the "coherent wave") and variance. In particular, one important result of multiple scattering theory is that $<s(\vec{r}, t)>$ follows Dyson's equation [13, 14]. If this equation can be solved, then the speed and attenuation can be inferred from a statistical description of the microstructure of any material. This was done for instance by Turner [15] in the framework of non-destructive characterization of polycrystals, where only one longitudinal mode was predicted and observed. The existence of two longitudinal modes within the framework of multiple scattering theory was reported by Cowan et al. [16]. They 
have carried out experiments showing that two-wave propaga-113 tion could occur in dense suspensions of plastic spherical scat-114 terers, and that the slower of the two waves resulted from resonant coupling between scatterers. Their theoretical approach ${ }^{115}$ was the multiple scattering theory, under the Coherent Potential ${ }^{116}$ Approximation [17], assuming the wavelength was larger than ${ }_{117}$ the scatterers size.

Actually, none of the above-mentioned theories is perfectly ${ }^{118}$ suited to wave propagation in cancellous bone. Unlike poly- ${ }^{119}$ crystals such as steel, cancellous bone has a solid and a fluid ${ }_{120}$ phase, showing strong variations for both density and elas- ${ }^{-121}$ tic moduli. Moreover, the typical dimensions of the hetero- ${ }^{-122}$ geneities are not small compared to the wavelength, at least in $_{123}$ the $\mathrm{MHz}$ range and above. One objective of this paper is to ex-124 amine in what respect some typical results of Biot's, Wood's $s_{125}$ and multiple scattering theories can be useful to understand ${ }_{126}$ wave propagation in cancellous bone. For instance Wood's $s_{127}$ model is very simple and purely empirical but we will see that ${ }_{128}$ in some cases it may suffice to predict the velocity. As to Biot's ${ }_{129}$ theory, apart from its inadequacy to describe scattering, it re- ${ }_{-130}$ quires many parameters that are difficult to measure in the case ${ }_{131}$ of cancellous bone, especially in vivo. As a consequence, multi- ${ }_{132}$ parameter fitting of experimental results is required, which im- ${ }^{-133}$ pairs the reliability of Biot's theory in the context of cancellous ${ }_{134}$ bone. To our knowledge Biot's theory was first developed for $_{135}$ isotropic media and does not clearly explain why the two waves ${ }_{136}$ are observed for an ultrasound propagation along the main ori- ${ }_{137}$ entation of the cancellous bone and not when the propagation is ${ }_{138}$ orthogonal [18]. Yet again, even though it fails to describe the ${ }_{139}$ attenuation and anisotropy, the velocities predicted by Biot can ${ }_{140}$ be in fairly good agreement with experimental observations. As $\mathrm{s}_{141}$ to multiple scattering theory, though it yields an exact equation ${ }_{142}$ for the coherent wave field, it is not ideal either because in or-143 der to derive practical results, at some stage an approximation ${ }_{144}$ has to be made, which necessarily limits the range of validity. In this paper, we will use the simplest of all multiple scattering models, known as the ISA (Independent Scattering Approximation), and examine its applicability to predict scattering losses 146 and dispersion in anisotropic porous structures.

For a better understanding of ultrasound propagation in can-148 cellous bone, we have chosen to begin with a numerical study, 149 which is particularly flexible. In the last ten years, numerical ${ }_{150}$ studies have been intensively used, both in real bone structures 151 derived from X-ray computed tomography $[19,20,21]$ or in nu-152 merically synthesized media [22]. Our approach here is based ${ }_{153}$ on the synthesis of numerical random and biphasic structures, 154 previously described in [23]: elliptic (2D) or ellipsoidal (3D) ${ }_{155}$ scatterers were randomly distributed in a fluid to form a slab of ${ }_{156}$ random medium. In this previous work, we had qualitatively ${ }_{157}$ studied the occurrence of the fast and slow waves depending on ${ }_{158}$ simple statistical medium parameters using a finite-difference ${ }_{159}$ time-domain (FDTD) tool $^{1}$ to simulate the propagation of elas- 160 tic waves. We observed similar results, at least qualitatively, in ${ }_{161}$ 2D and in 3D. Four conditions were necessary to observe the ${ }_{162}$ two waves in our simple models:

${ }^{1}$ http://www.simsonic.fr
- a certain range of solid fraction (around 30\% to $70 \%$ for our model)

- a propagation parallel to the main orientation (i.e. in the direction of the long axis of the scatterers)

- the elastic nature of the solid phase

- enough connectivity of the solid matrix along the direction of propagation

The first two points are consistent with previous observations in real cancellous bone [11, chap.11], justifying a posteriori the relevance of our simplified model as a first step to study ultrasound propagation in trabecular bone. The last two points, on the contrary, could only have been studied numerically, and were consistent with the hypothesis that the fast wave results from a guiding through the solid matrix, whereas the slow wave is traveling predominantly through the fluid.

In the present article, velocities and attenuation coefficients of the coherent waves are measured in 3D samples, whether one or two waves are observed. Measurements methods are detailed in Sec.2.2. FDTD simulation results are confronted to the Independent Scattering Approximation (see Sec.2.3) and Biot's theory. Comparisons are presented in Sec.3, when one (3.1) or two waves (3.2) are observed. Sec.3.3 presents a different approach based on wave propagation from a pointlike source in 2D media, in order to observe the progressive transition from one to two waves. Finally, Sec.4 investigates the nature of the two waves by different methods. First, limit cases are studied by observing the two waves when the fluid phase is replaced by vacuum or when the solid matrix is perfectly rigid. In these cases propagation occurs only in the solid (respectively fluid) phase, giving interesting clues on the propagation paths for the fast and slow waves. Finally, we compare the phase shifts between the simulated fast and slow waves to Biot's theory.

\section{Material and Methods}

\subsection{Numerical simulations}

In a previous paper [23] we introduced numerical models generated by a Monte Carlo method: ellipses (2D) or ellipsoids (3D) of solid aligned along the same direction were added progressively in water (overlap was allowed), in order to obtain anisotropic and biphasic maps with given solid fractions. Examples of 3D maps can be seen on figure 1. The half lengths of the principal axes of the scatterers (i.e. the ellipsoids) were $a=350 \mu m, b=c=50 \mu m$ (note that $c$ does not exist in 2D). The material properties chosen for the solid part were those of typical compact bone, compressional velocity $c_{L}=4 \mathrm{~mm} \cdot \mu \mathrm{s}^{-1}$, shear velocity $c_{T}=1.8 \mathrm{~mm} \cdot \mu \mathrm{s}^{-1}$ and mass density $\rho_{s}=1850 \mathrm{~kg} \cdot \mathrm{m}^{-3}$. The background medium had the properties of water, $V_{\text {fluid }}=1.5 \mathrm{~mm} . \mu \mathrm{s}^{-1}$ and mass density $\rho_{f}=1000 \mathrm{~kg} . \mathrm{m}^{-3}$. Ultrasound propagation was simulated by a FDTD software, SimSonic, developed by E. Bossy [24] and freely available for download on-line ${ }^{2}$. Stresses and particle velocities can be obtained at each point of the medium. Perfectly

${ }^{2}$ http://www.simsonic.fr 

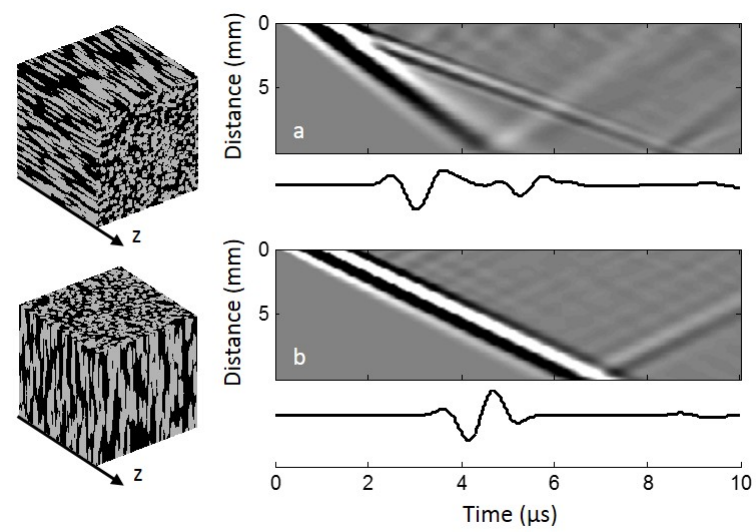

Figure 1: Left: Two typical 3D maps, both with a 50\% solid fraction (gray). The21 propagation direction is along the $\mathrm{z}$ axis. (a) Time-distance diagram and corre- ${ }_{212}$ sponding time trace (taken at a $6 \mathrm{~mm}$ propagation distance) obtained when the ultrasound propagation is along the main direction. Two wavefronts are clearly ${ }^{21}$ distinguished. This simulation will be referred to as the "reference simulation". (b) Time-distance diagram and corresponding time trace (taken at a $6 \mathrm{~mm}$ propagation distance) obtained when the ultrasound propagation is perpendicular the main direction. Only one wave is observed. matched layers (PML) were placed at the edges of the sample in the direction of propagation, while perfectly reflecting walls were placed in the transverse direction, so that the samples can be considered as semi-infinite slabs. The ultrasound pulses considered in the simulations had a central frequency of $1 \mathrm{MHz}$. The grid size was 10 micrometers in every directions, which corresponds to $1 / 150^{\text {th }}$ of the wavelength in the fluid. Taking advantage of the flexibility of numerical methods, we recorded the signals transmitted inside the samples for different depths within the propagation medium, in order to obtain full time-distance diagrams where the signal amplitude can be read as a function of time and propagation depth. This type of representation, not accessible experimentally where only time traces can be retrieved outside the sample, makes it easier to determine whether one or two waves are propagating. This is illustrated in Fig.1: in the case of a propagation along the main direction, two waves are clearly observed (top) whereas only one wavefront appears in the case of a propagation perpendicular to the main direction, i.e. along the long axis of the ellipsoids (bottom). The transmitted signal is integrated over the whole transverse plane, for estimation of the coherent wave, assuming ergodicity. Due to the reflecting walls, the resulting signal is analogous to what would be measured in an ideal experiment with an in- ${ }^{214}$ finite planar transducer placed within a semi-infinite scattering ${ }^{215}$ slab. More details on both the numerical samples and the $\operatorname{sim}^{-216}$ ulations are available in [23]. In the rest of the present article, ${ }^{217}$ the simulation of propagation along the main direction in a bi- ${ }^{218}$ nary medium made of bone and water with a $50 \%$ solid fraction ${ }^{219}$ (Fig.1a) will be referred to as the "reference simulation".

\subsection{Velocities and attenuation coefficients measurements}

As the emitted signal is a pulse centered at $1 \mathrm{MHz}$ with $-6 \mathrm{~dB}$ bandwidth of around $100 \%$, we limited our study to the 224 $0.5 \mathrm{MHz}-1.5 \mathrm{MHz}$ frequency range. Theoretically the coher-225 ent wave is obtained through ensemble averaging of the signals propagated through an infinite number of realizations, and is therefore only estimated here. As a consequence there is still an incoherent part remaining in the studied signal, mostly arriving after the ballistic wave(s). In order to remove it, the fast and slow waves were separated using a combination of rectangular and Hann windows, to avoid as much as possible the creation of disruptive frequencies brought by sharp cutting.

In a first step we focused on obtaining velocities and attenuation coefficients from the 3D numerical simulations. As detailed above, transmitted signals for different propagation distances were recorded and stored in a time-distance matrix $s(t, z)$. Performing Fast Fourier Transforms (FFT) of each column provides $\tilde{s}(\omega, z)$, a matrix where each row corresponds to $\tilde{s}_{\omega}(z)$ the signal as a function of propagation distance, at a given angular frequency $\omega$. Phase velocity and attenuation coefficient were respectively obtained as follows:

- by taking the unwrapped phase angle $\varphi_{\omega}(z)$ of $\tilde{s}_{\omega}(z)$ we obtained

$$
\varphi_{\omega}(z)=\arg \left(\tilde{s}_{\omega}(z)\right)=\varphi_{\omega}(0)+k z
$$

so $\varphi_{\omega}(z)$ is linear, with a slope equal to $k(\omega)$ giving easy access to the phase velocity $v(\omega)$ :

$$
v(\omega)=\frac{\omega}{k(\omega)}
$$

- by taking the modulus of $\tilde{s}_{\omega}(z)$ and assuming an exponential decrease of the signal, one obtains the following expression involving the attenuation coefficient $\gamma(\omega)$ :

$$
\left|\tilde{s}_{\omega}(z)\right|=e^{-\gamma(\omega) z}
$$

$\gamma$ is then obtained by a linear fit of $\ln \left|\tilde{s}_{\omega}(z)\right|$ with z. Note that we chose to represent the attenuation coefficient for the amplitude $\gamma$ as it is usually used in the field of bone quantitative ultrasound [11]. In the multiple scattering community, one usually refers to the scattering mean free path $l_{e}$, defined as the decay length for the intensity of the coherent field. The two parameters are simply related:

$$
l_{e}=\frac{1}{2 \gamma}
$$

Frequency-resolved measurements of attenuation and velocities were obtained for the reference simulation (as exposed in Sec.3.2). However Eq.2 is inapplicable when the two wavefronts are not clearly separated. In such cases, fast and slow waves velocities were estimated through time-of-flight measurements, by tracking the first minimum of each wave in the time-distance diagrams, thus losing the frequency-dependence.

\subsection{Independent Scattering Approximation (ISA)}

In a random scattering medium the coherent field is the solution of Dyson's equation [13]. The key element in Dyson's equation is the so-called "self-energy" which wraps up all possible multiple scattering terms. The self-energy can be written 
Here, the numerical samples can be seen as a random ar-253 rangement of identical scatterers in a lossless fluid with velocity ${ }_{254}$ $c_{0}$ (wavenumber $k_{0}=\omega / c_{0}$ ). In that simple case, from a physi-255 cal point of view the ISA amounts to considering that the scat-256 terers positions are uncorrelated, and that the wave never loops 257 back to a scatterer that has already been visited [25]. Under 258 this assumption, the self-energy only depends on the scatterers259 concentration $n$ and the scattering properties of one individual ${ }_{260}$ scatterer, particularly its angular directivity pattern $f(\theta)$. Then Dyson's equation has an analytical solution, and the coherent 261 wavefield is characterized by a dispersion equation $k_{e f f}(\omega)$, with $k_{\text {eff }}$ the complex-valued "effective wave number". In other words, on average the effect of multiple scattering is to modify the speed as well as the attenuation of the medium, since $k_{\text {eff }}$ is a complex number, unlike $k_{0}$. Under the ISA, the effective wave number $k_{e f f}$, and as a consequence velocity and attenuation, can be estimated from the density of scatterers $n$, the speed of sound in the surrounding fluid $c_{0}$ and the forward-scattered pressure $\tilde{\psi}_{\text {scat }}$, with

$$
\tilde{\psi}_{\text {scat }}(\theta=0, r)=\frac{e^{i k_{0} r}}{r} f(\theta=0)
$$

This last parameter is obtained at a distance $r$ in the direction of incidence $(\theta=0)$ when one single scatterer immersed in the fluid is insonified by a plane wave. The resulting dispersion relation is:

$$
k_{e f f}^{2}=k_{0}^{2}+4 \pi n f(\theta=0)
$$

where $n$ is the solid fraction $\Phi_{s}$ divided by the volume of a ${ }^{264}$ single scatterer ( $a, b$ and $c$ are the half axes defined in Sec. 2.1) $)^{265}$

$$
n=\frac{\Phi_{s}}{\frac{4}{3} \pi a b c}
$$

The forward scattered pressure $\tilde{\psi}_{\text {scat }}(\theta=0, r)$ and $f(\theta=0)_{270}$ are obtained numerically by a simple FDTD simulation where 271 a plane wave is emitted in a medium containing only one scat-272 terer surrounded by water. The medium is $8 \mathrm{~mm}$ thick in the ${ }_{273}$ propagation direction and $16 \mathrm{~mm} \times 16 \mathrm{~mm}$ large in the trans-274 verse directions. The center of the scatterer is just ahead of the 275 emitting boundary at a $500 \mu \mathrm{m}$ depth, centered in each trans-276 verse direction. The signal is recorded at a $7.5 \mathrm{~mm}$ distance ${ }_{277}$ ahead of the center of the scatterer. We chose the same grid $_{278}$ step size as for the random media simulations to account for the 279 discretization effects at the border of the ellipsoid.

280

For the single scatterer simulation, boundary conditions were ${ }_{281}$ chosen strictly similar to those of the random media simulation ${ }_{282}$ (PML in the direction of propagation, symmetry conditions in ${ }_{283}$ transverse directions) to ensure a perfect incoming plane wave. 284 The forward scattered signal had to be windowed to limit the 285 contribution of image scatterers due to the symmetry condi-286 tions. As a reference, the same simulation was run with no 287 scatterer. The resulting field $\psi_{0}(\theta=0, r, t)$ was then subtracted ${ }_{288}$ from the total field in order to obtain the forward scattered field $\psi_{\text {scat }}(\theta=0, r, t)$. Finally, the Fourier transform of the scattered field was normalized by that of the incident wave to obtain $\tilde{\psi}_{\text {scat }}(\theta=0, r)$ which comprises only the frequencies in the bandwidth of the incident pulse i.e., from $0.5 \mathrm{MHz}$ to $1.5 \mathrm{MHz}$.

It should be noted that Eq. 6 implies that there is only one effective number. Therefore intrinsically the ISA only predicts the existence of one longitudinal mode, with a velocity and an attenuation different from that of the fluid. However even when two waves are observed, it may be interesting to compare the velocity and attenuation predicted by the ISA with the simulated results.

\subsection{Some predictions of Biot's theory}

Biot's theory [6, 7] was originally developed for the study of ultrasound propagation in porous, isotropic rocks, with a low frequency assumption. Various groups have used Biot's framework in other fields of application and with additional hypotheses, providing in some cases the assessment of wave velocities from only a few parameters. For example, in the limit where the porous frame is much stiffer than the fluid, Johnson [26] gives simple relations (see Appendix A) from which we can derive the velocities of the fast and slow waves:

$$
\begin{aligned}
& V_{\text {fast }}=\frac{V_{d r y}^{L}}{\sqrt{1+\frac{\Phi_{f} \rho_{f}}{\Phi_{s} \rho_{s}}\left(1-\frac{1}{\alpha}\right)}} \\
& V_{\text {slow }}=\frac{V_{\text {fluid }}}{\sqrt{\alpha}}
\end{aligned}
$$

where $\Phi_{f}$ and $\Phi_{s}$ are the fluid and solid fractions, $\rho_{f}$ and $\rho_{s}$ the fluid and solid densities, $V_{\text {fluid }}$ the speed of sound in the filling fluid. $V_{d r y}^{L}$ is the longitudinal speed of sound in the dry sample, i.e. the velocity when the fluid is replaced by vacuum, a situation which is easy to simulate numerically. Finally $\alpha$ is the geometric tortuosity, which is particularly difficult to assess in porous media and is by definition independent of material properties but depends on the micro-architecture.

Note that there is no frequency dependence in Eq.8 and 9, which is consistent with the use of time-of-flight measurements to estimate velocities from experimental results.

Biot's framework is also used in this study to gain insight on the origin of the fast and slow waves. One of the main conclusions of Biot's theory is that the fluid and the solid move either in phase (fast wave) or out of phase (slow wave) [6]. In order to check this prediction, the transverse plane is divided in two regions corresponding to solid and fluid zones respectively. The particle velocity is integrated separately in the two areas, as if the receiving transducer was only in contact with the fluid or with the solid. Then we can examine whether the resulting coherent waves in the fluid and in the solid exhibit a particular phase shift. This peculiar prediction cannot be verified experimentally, unless we could have a point-like transducer deep inside the sample measuring displacements in the fluid and in the solid. But numerical simulations make that measurement possible. Results are shown in Sec.4.2, for two different solid fractions (50\% and $70 \%)$. 


\section{From one to two waves}

\subsection{One wave}

In a first step, we compare the frequency-dependence of attenuation coefficient and velocity (measured as described in Sec.2.2) to ISA predictions, in samples where only one compressional wave could be observed. As shown in [23] and Fig.1, this occurs when the ultrasound propagation is perpendicular to the main direction of the samples. The velocities and attenuation coefficients are plotted in Fig.2 for various solid fractions: $10 \%, 30 \%$ and $50 \%$.

As expected, the performance of ISA strongly depends on the density of scatterers. For a low solid fraction (10\%) both velocities and attenuation coefficients are well predicted by the ISA. The discrepancy increases with solid fraction. These results are in agreement with the fact that the ISA is a first-order approximation, which naturally fails as the solid fraction increases. Another interesting point is that a negative dispersion was observed for the three different bone fractions. In fact, the observed linear relationship between velocity and frequency, with a negative slope, was well predicted by the ISA. This negative dispersion is of particular interest because it has also been observed experimentally in cancellous bone [27]. The velocity ${ }^{322}$ increases, as expected, with the solid fraction. In the simple 23 case of one wave propagation, Wood [5] theory could be used ${ }^{324}$ for estimating porosity based on velocity measurements. Ac-325 cording to Wood, the compressional wavespeed of an effective 326 medium depends on 5 parameters: the fluid and solid densities 327 $\rho_{f}$ and $\rho_{s}$, the fluid and solid bulk moduli $K_{f}$ and $K_{s}$ and the fluid and solid fractions $\Phi_{f}$ and $\Phi_{s}$ :

$$
V_{\text {Wood }}=\sqrt{\frac{K_{f} K_{s}}{\left(\Phi_{f} K_{s}+\Phi_{s} K_{f}\right)\left(\Phi_{s} \rho_{s}+\Phi_{f} \rho_{f}\right)}}
$$

Fig. 3 compares the velocities obtained in our samples (simplyзз deduced from time-of-flight measurements in the time-distance $з з$ diagrams) with the one predicted by Wood in a range of solid ${ }_{334}$ fraction going from 0 to 0.5 .

There is a good agreement between the velocities measured ${ }_{336}$ with the simulations and predicted by Wood, especially for aз37 low solid fraction. In fact as seen in Fig. 2 the higher the solid ${ }_{338}$ fraction the higher the dispersion, so for higher solid fractions, 339 the time-of-flight methods for velocity measurements probably 340 become biased. However this result shows that in this configu-341 ration (propagation perpendicular to the main direction) micro-342 architecture parameters other than porosity do not seem to play 343 a role in the velocities. In some cases where only one com-344 pressional wave was observed, Wood and Biot theories were 345 found to yield similar values for the velocity [28]. As to the 346 attenuation coefficient, it is worth noticing that the observed at-347 tenuation coefficient shows a power-law dependency with fre- 348 quency, with characteristic exponents of 3.4, 2.5 and 2.1 for $_{349}$ $10 \%, 30 \%$ and $50 \%$ porosity. When increasing the solid frac-350 tion, the scatterers are more likely to overlap and create struc-351 tures significantly larger than the wavelength. The decay of ${ }_{352}$ the characteristic exponent is therefore consistent with predic-353 tions from scattering theories, where the attenuation coefficient ${ }_{354}$

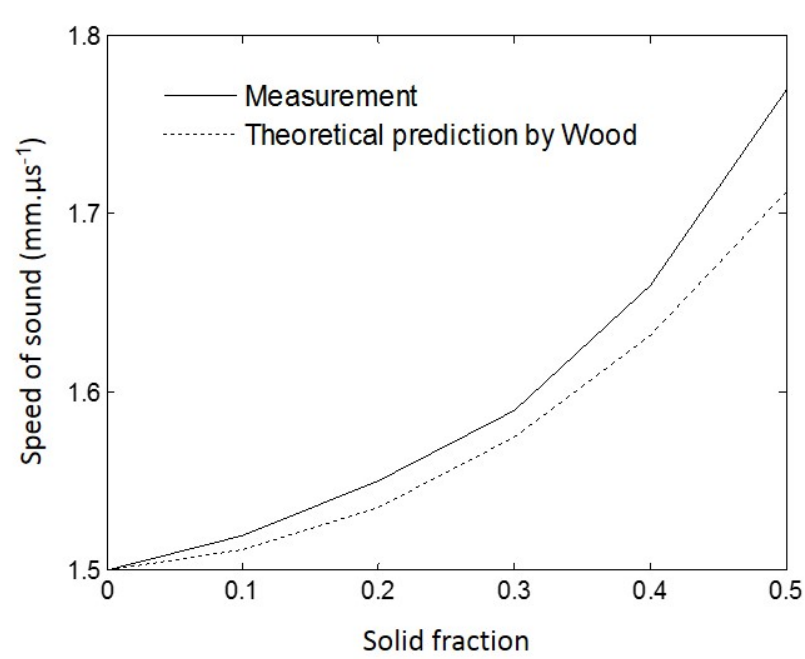

Figure 3: Speed of sound as a function of solid fraction (from 0 to 0.5 ) measured from time-of-flight measurements in the time-distance diagrams and predicted by Wood, using Eq.10

varies as $\omega^{4}$ in the very low frequency regime and as $\omega^{2}$ in the high-frequency regime. But this is very different from the usual linear dependence with frequency which has often been reported in cancellous bone [29, 30]. This indicates that our model samples exhibit some, but not all of the features typical of porous bone.

\subsection{Two waves}

We now move to the case where two compressional waves propagate. Previous work by our group showed that the two waves could be observed in the case of an ultrasound propagation along the main direction and for an appropriate range of solid fraction, from $30 \%$ to $70 \%$ [23] (Fig.1). The two-wave configuration is a little more difficult to study because it requires the two waves to be separated, in order to apply the routine described in Sec.2.2. We therefore limited the study to the case of a $50 \%$ solid fraction, where the two waves were found to be best separated. In addition, the propagation depth was increased to $20 \mathrm{~mm}$ in order to facilitate the distinction between the slow and the fast wave. The simulation results show that the two waves were conveniently separated for depths ranging between $6.7 \mathrm{~mm}$ and $11.7 \mathrm{~mm}$, without being too strongly attenuated by scattering or polluted by reflected waves from the sample boundary. Hann windowing was used to isolate the slow wave. Fig. 4 shows the resulting attenuation coefficients and velocities of the fast and slow waves, as well as the ISA predictions.

ISA only predicts one wave, as discussed in Sec.2.3. Furthermore the velocity predicted by ISA (around $1.5 \mathrm{~mm} . \mu \mathrm{s}^{-1}$ ) was far from the measured velocities for the fast (around $2.6 \mathrm{~mm} . \mu \mathrm{s}^{-1}$ ) and slow (around $1.3 \mathrm{~mm} . \mu \mathrm{s}^{-1}$ ) waves. This can be explained by the fact that the Independent Scattering Approximation sees the medium as a perturbation of the surrounding fluid (water with a speed of sound of $1.5 \mathrm{~mm} . \mu \mathrm{s}^{-1}$ ). Here, 

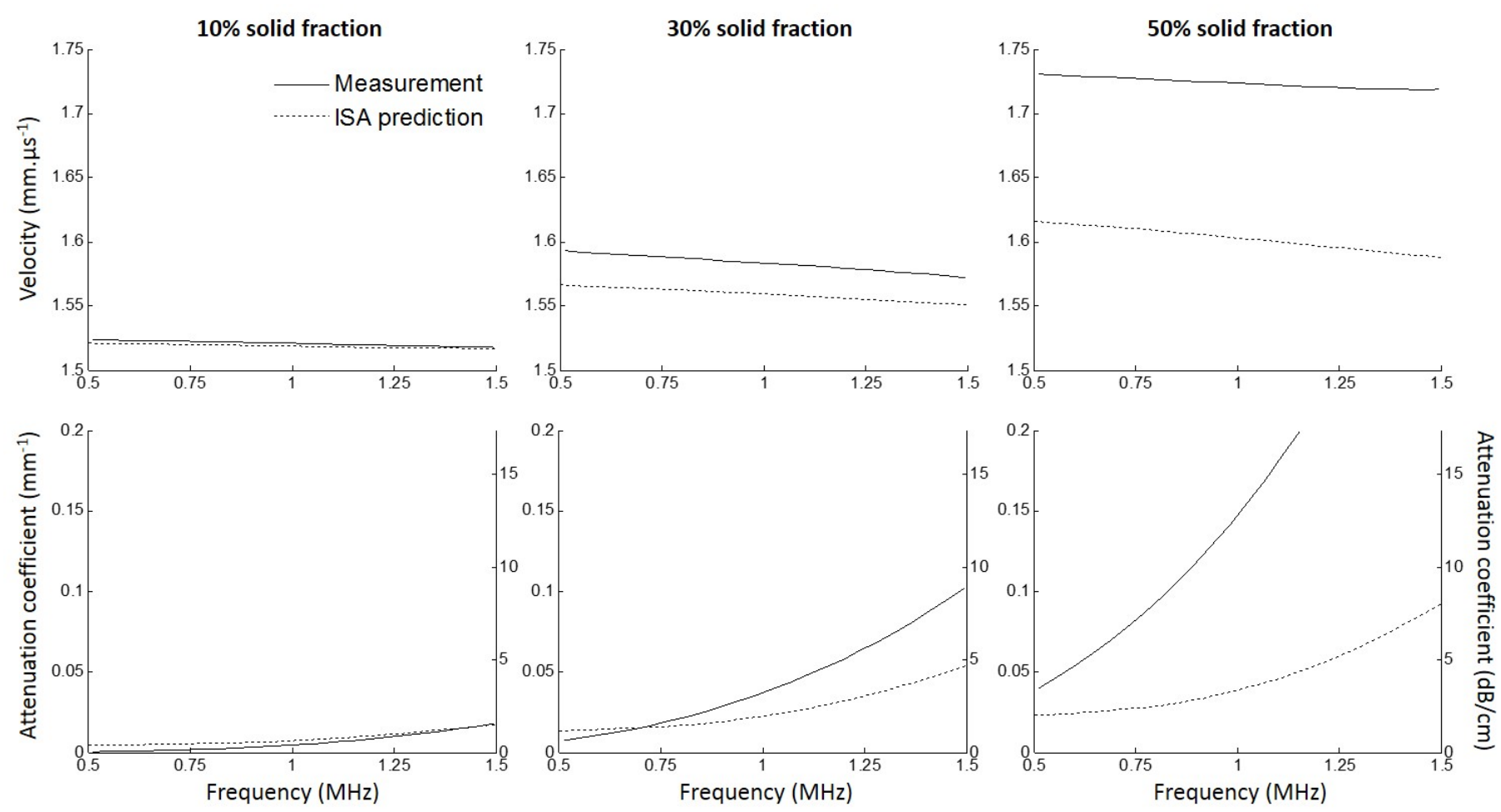

Figure 2: Velocities (top) and attenuation coefficients (bottom) measured in three different samples with a 10\% (left), 30\% (center) and 50\% (right) solid fraction. For all three cases, ultrasound propagation was set perpendicular to the direction of anisotropy so that only one wave could be observed. The three velocity measurements exhibit a linear dependence of frequency with a negative dispersion (the slopes are $-0.006 \mathrm{~mm}$ for the $10 \%$ solid fraction, $-0.02 \mathrm{~mm}$ for the $30 \%$ one and $-0.013 \mathrm{~mm}$ for the $50 \%$ one) quite well predicted by ISA (respectively $-0.005 \mathrm{~mm},-0.016 \mathrm{~mm}$ and $-0.029 \mathrm{~mm}$ ).

the bone fraction is far beyond the limit of use of this theory ${ }^{3}$ as $_{377}$ already discussed in the previous subsection, but this is require ${ }_{378}$ to observe two separable waves for our numerical samples [23].379 Still, it is interesting to notice that fast and slow waves attenua-380 tion coefficients seem of the same order of magnitude. It should ${ }_{381}$ be pointed out that Biot's theory predicts no attenuation (loss-382 less fluid), whereas numerical results clearly show that both fast 383 and slow waves undergo a strong attenuation due to scattering. The observed attenuation is significantly stronger than what the 385 ISA predicts. It is also interesting to notice that both waves 386 show a slight positive dispersion. This is consistent with previ-387 ous observations on cancellous bone, where no negative disper-388 sion was observed when the two waves were clearly separated $d_{389}$ [1][11, chap.5]. Finally, note that the slow wave velocity is slower than the speed of sound in water, which is in agreement ${ }^{390}$ with Eq.9, as tortuosity is real and greater than unity.

\subsection{Source point}

Let us now consider a different approach where a source point is placed at the center of the map (to avoid a possible nu- ${ }_{396}$ merical issue, we made sure this point lied in the fluid phase). Propagation can be studied along all directions simultaneously ${ }_{398}$ giving much more information than the previous plane wave ${ }_{399}$ section of a single scatterer. The high-frequency limit for $\sigma$ is twice the geo- 402 metric cross-section simulations. In the point-source configuration, the coherent wave could not be estimated by spatial averaging anymore, and ensemble averaging over 50 realizations of the random medium was performed. As the computational cost is much higher, the simulations were carried out only in $2 \mathrm{D}$. It has previously been shown that qualitative results were similar in 2D and in 3D [23]. Each map is $15 \mathrm{~mm}$ by $15 \mathrm{~mm}$ with a $5-\mu m$ grid step. In order to account for the geometrical decay introduced by propagation from a source point, the signals were multiplied by $\sqrt{r}, r$ being the distance from the point of observation to the source point. Fig. 5 shows snapshots of the propagation after averaging over the 50 realizations. Clearly, the incoherent contribution has not yet been completely cancelled out.

Fig. 5 shows a strong anisotropy of the propagation through the random samples. In the main direction (horizontal) two wavefronts can be distinguished, even though the ratio of coherent to incoherent wave amplitude would have benefited from averaging over a larger number of realizations. This result is in agreement with the previous observations. If we continuously rotate to the case where the propagation is perpendicular to the main axis, the fast and slow waves velocities are getting closer until only one wave can be distinguished. This observation rules out the possibility that the velocity of the slow wave continuously drops to zero when the propagation direction changes from parallel to perpendicular to the main axis. It also raises questions about the phenomenon actually taking place when only one wave can be observed: two waves could 


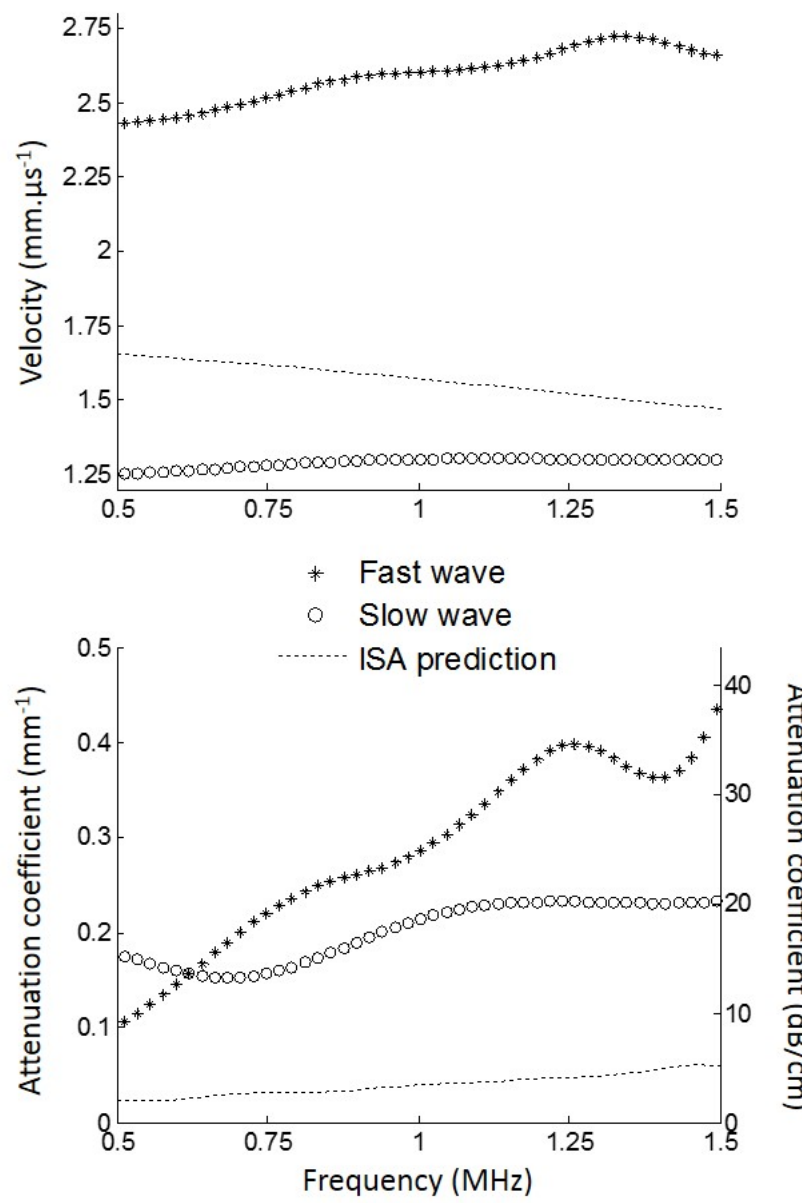

Figure 4: Velocities (top) and attenuation coefficients (bottom) of the fast and ${ }^{41}$ slow waves, measured in a 50\% solid fraction sample in the case of ultrasound propagation along the main direction. Confrontation with the ISA prediction 412
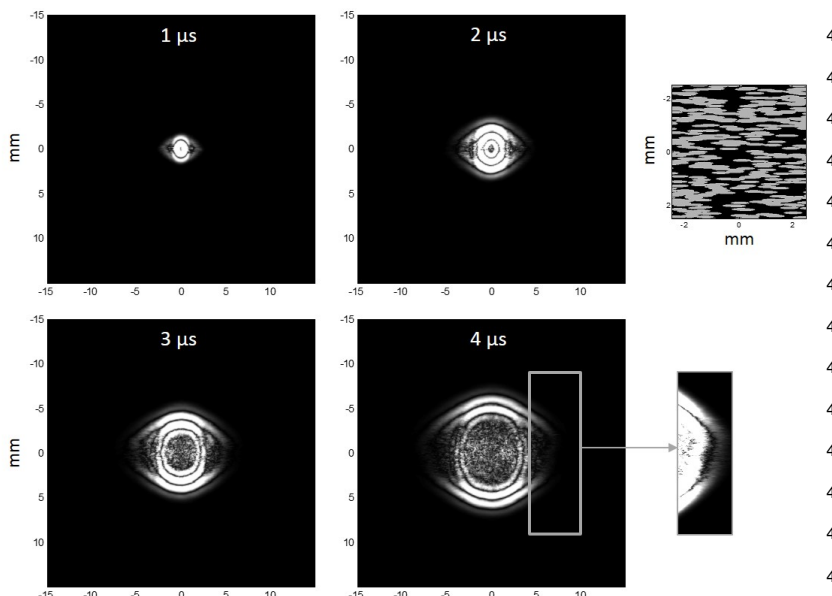

$\mathrm{mm}$

$\mathrm{mm}$
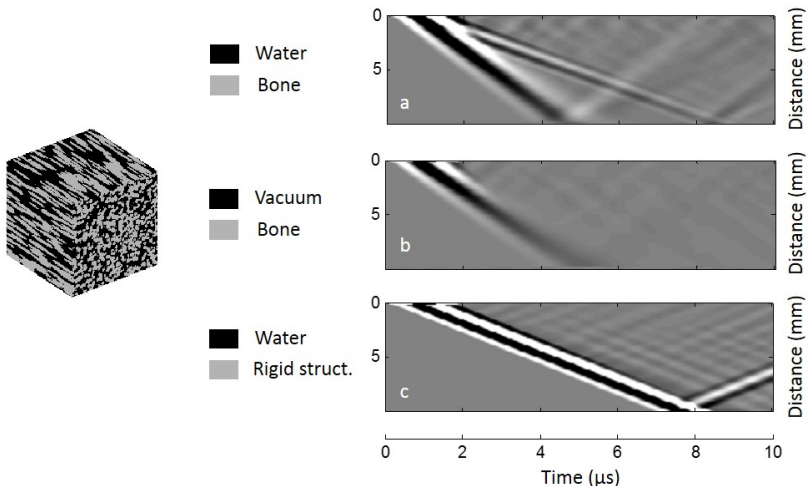

Figure 6: Left: The same 3D arrangement (collection of solid ellipsoidal scatterers, with a $50 \%$ solid fraction, propagation along the main direction) was used for the three simulations. Grey: solid phase. Black: fluid. (a) Reference simulation (same as in Fig.1a), Solid: Bone. Fluid: Water. Two wavefronts are clearly distinguished. (b) Solid: Bone. Fluid: Vacuum. Only one wavefront is observed, with a velocity (around $2.5 \mathrm{~mm} . \mu \mathrm{s}^{-1}$ ) close to that of the fast wave (around $2.6 m m . \mu s^{-1}$ ) in (a). (c) Solid: Infinite density. Fluid: Water. Only one wave is observed, with a velocity (around $1.45 \mathrm{~mm} . \mu \mathrm{s}^{-1}$ ) close to that of the slow wave (around $1.3 \mathrm{~mm} . \mu \mathrm{s}^{-1}$ ) in (a)

actually be propagating with very close velocities. The negative dispersion obtained from the propagation of a single wave, perpendicular to the main orientation of the scatterers could be re-interpreted in the light of this last result. If two waves are actually propagating with close velocities, the corresponding pulses could be interfering, leading to an apparent negative dispersion, as observed by Anderson et al. [31].

\section{Insights on the nature of the two waves}

\subsection{Limit cases}

One great advantage of numerical simulations is the possibility to fully control the properties of the simulated medium. Fluid or solid properties were modified in the reference simulation, where two waves could be observed. First, water was replaced by vacuum (density and elastic constants were set equal to zero). Ultrasound propagation could therefore only occur in the solid frame. Second, in another simulation, the solid phase (which had initially the properties of bulk bone) was turned into a perfectly rigid frame, forcing the ultrasound propagation to occur only through water. Those two cases, which can be seen as limit cases when the density of the fluid (respectively solid) phase reaches zero (respectively infinity) would have been nearly impossible to study experimentally. The results are compared with that of the reference case in Figure 6.

When ultrasound propagation was only allowed in one of the two phases, only one wavefront was observed, whereas there were clearly two distinguishable wavefronts in the reference simulation. Furthermore, the velocity of the wave when the propagation occured only through the solid (respectively fluid) was close to that of the fast (respectively slow) wave in the reference case. This result suggests that the fast wave travels mostly through the solid frame, and the slow wave mostly through the fluid, in accordance with previous results [23]. It

Figure 5: Estimation of the coherent wave propagation from a source point in ${ }_{430}$ a 2D anisotropic porous media with a 50\% solid fraction. Left: 4 snapshots, taken at $1 \mu s, 2 \mu s, 3 \mu s$ and $4 \mu s$. Top right: detail of one realization showing the direction of the ellipses. Bottom right: A part of the fourth snapshot with a ${ }^{432}$ saturated contrast to better observe the fast wave. Two waves are observed for a 433 propagation parallel to the main axis, only one is observed for a perpendicular ${ }_{434}$ propagation. 


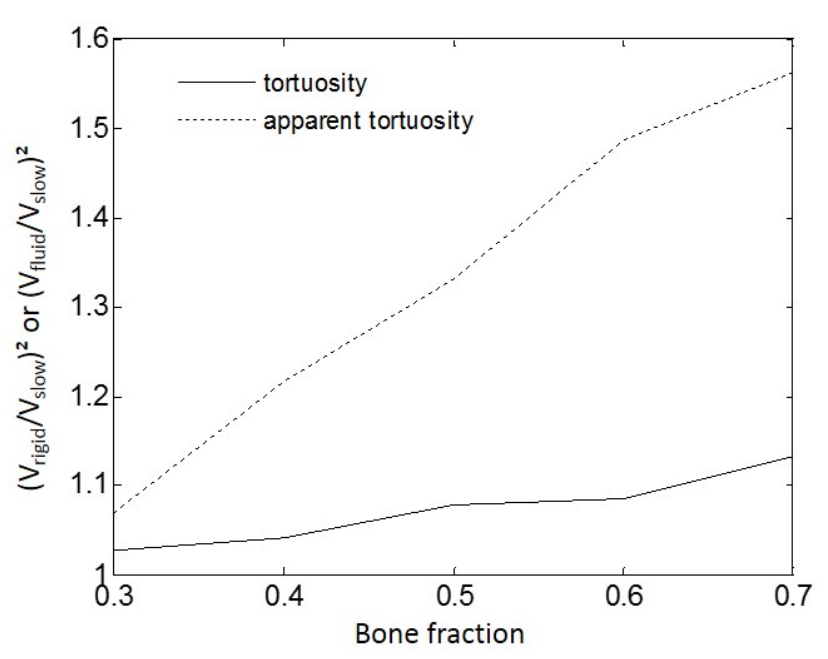

Figure 7: Tortuosity (deduced from rigid frame velocity) and apparent tortusoity (deduced from slow wave velocity) as a function of bone fraction is interesting to notice that the speed $V_{\text {rigid }}$ of the wave propagating only in water with a perfectly rigid frame (Fig.6c), around $1.45 \mathrm{~mm} \cdot \mu \mathrm{s}^{-1}$, is lower than the speed of sound in water $\left(V_{\text {fluid }}=1.5 \mathrm{~mm} \cdot \mu \mathrm{s}^{-1}\right)$ but larger than $V_{\text {slow }}$ in Sec.3.2, around $1.3 \mathrm{~mm} \cdot \mu s^{-1}$. These values gives interesting information. First, $V_{\text {rigid }}<V_{\text {fluid }}$ and $V_{\text {slow }}<V_{\text {fluid }}$ which accounts for the tortuos-477 ity of propagation paths in water. However, as $V_{\text {rigid }} \neq V_{\text {slow }}$ the 478 slow wave formula (Eq.9) described in Sec.2.4 does not seem to 479 hold. This suggests that the mechanical properties of the skele-480 ton influence the slow wave velocity, which invalidates the stiff ${ }_{481}$ frame hypothesis. If tortuosity were to be estimated from veloc-482 ity measurements, one should use $V_{\text {rigid }}$ and Eq.9. Still, from ${ }_{483}$ an experimental and practical point of view, we could define ${ }_{484}$ an "apparent tortuosity" from Eq.9 using $V_{\text {slow }}$, which, unlike 485 $V_{\text {rigid }}$, is accessible from real experiments. Figure 7 shows the ${ }_{486}$ tortuosity and "apparent tortuosity" obtained at various solid fractions, ranging from $30 \%$ to $70 \%$. Tortuosity and "apparent 487 tortuosity" are both confined between 1.03 and 1.56 and seem to ${ }_{488}$ converge to 1 at low solid fraction. The difference is at its highest for high solid fractions (low porosity). Hence, in the case of actual cancellous bones where porosity lies between $75 \%$ and $_{491}$ $95 \%$, the difference may not be so significant. This could open ${ }_{492}$ an interesting perspective: the possibility to measure tortuosity ${ }_{493}$ from the slow wave velocity in highly porous bones.

To conclude this subsection we confront the velocity mea- ${ }_{495}$ sured in the reference simulation to the prediction of Eq.8. ${ }_{496}$ To do so we extract $V_{d r y}^{L}$ from the simulation where the fluid ${ }_{497}$ is turned into vacuum (Fig.6b) by a time-of-flight measure- ${ }_{498}$ ment. Then the only missing parameter is the geometric tor- ${ }_{499}$ tuosity $\alpha$. As we have seen just before there are two differ- ${ }_{500}$ ent values for this parameter. The stiff frame assumption im- ${ }_{501}$ plies that $\left(K_{d r y} \gg K_{f}\right.$ and $\left.N_{d r y} \gg K_{f}\right)$ with $K_{d r y}$ and $N_{d r y 502}$ the bulk and shear moduli of the dry sample [26]. These two parameters can be retrieved from $V_{d r y}^{L}=2.5 \mathrm{~mm} . \mu \mathrm{s}^{-1}$ and $\mathrm{to}_{504}$ $V_{d r y}^{T}=0.8 m m . \mu s^{-1}$ i.e., the transverse speed of sound in the dry $y_{505}$ sample (see Appendix A) that we can also calculate thanks to506 the propagation of a plane shear wave. We find $K_{d r y}=5.2 \mathrm{GPa}$ and $N_{d r y}=0.6 \mathrm{GPa}$, to be compared to $K_{f}=2.25 \mathrm{GPa}$. This confirms that the stiff frame assumption does not hold, and explains why the apparent tortuosity differs from the actual tortuosity.

However, using the fast wave formula (Eq.8) and knowing that both values of tortuosity remain close to 1 and that $\rho_{s}$ is almost twice $\rho_{f}$, one obtains:

$$
\frac{\Phi_{f} \rho_{f}}{\Phi_{s} \rho_{s}}\left(1-\frac{1}{\alpha}\right) \ll 1
$$

and as a consequence

$$
\begin{aligned}
V_{\text {fast }} & =\frac{V_{d r y}^{L}}{\sqrt{1+\frac{\Phi_{f} \rho_{f}}{\Phi_{s} \rho_{s}}\left(1-\frac{1}{\alpha}\right)}} \\
& \approx V_{d r y}^{L}=2.5 \mathrm{~mm} . \mu \mathrm{s}^{-1}
\end{aligned}
$$

This explains the observations made from figure 6, and agrees with the calculation with either one or the other value for the tortuosity:

$$
\begin{aligned}
V_{\text {fast }}^{\text {tort }} & =2.5 \mathrm{~mm} \cdot \mu \mathrm{s}^{-1} \\
V_{\text {fast }}^{\text {app tort }} & =2.3 \mathrm{~mm} \cdot \mu \mathrm{s}^{-1}
\end{aligned}
$$

Both values are close to the actual measured fast wave velocity (2.6 mm. $\mu \mathrm{s}^{-1}$ deduced from the value at $1 \mathrm{MHz}$ in Fig.4).

Although the stiff frame assumption might not hold here, it appears that this does not affect the prediction of $V_{\text {fast }}$ using Biot's theory. Biot's theory therefore gives correct orders of magnitude for the fast wave velocity in our anisotropic porous models, even beyond the frequency limit of this theory. It does not, however, predict the positive dispersion of the two waves, or the negative dispersion of the single wave, and does not account for scattering losses.

\subsection{Phase shift of the fast and slow waves}

One of the most striking results in Biot's model is that the fast and slow waves are associated respectively to in-phase and outof-phase displacements of the fluid and solid skeleton. Interestingly, numerical simulation gives us the possibility to check if the average motion within the medium follows this peculiar behavior. As a last point of this paper, we studied the phase shifts between the displacements in each phase (solid or fluid) according to the method explained in Sec.2.4. Note that we have tested both to integrate particle velocities only at the interfaces or over the whole phases and it has shown very little difference, for both $70 \%$ and $50 \%$ solid fractions. As a consequence only the integrations over the whole phases are represented in Fig.8.

In each case, the phase shift was close to but not exactly that predicted by Biot: the observed phase shifts are $10^{\circ}(70 \%$ solid fraction) and $19^{\circ}$ (50\% solid fraction) for the fast wave and respectively $174^{\circ}$ and $163^{\circ}$ for the slow wave. In a previous work [23] it was suggested that the physical origin for the occurrence of two waves could be that the bone trabeculae (or the connected ellipsoids) act as waveguides. This is the reason why we 

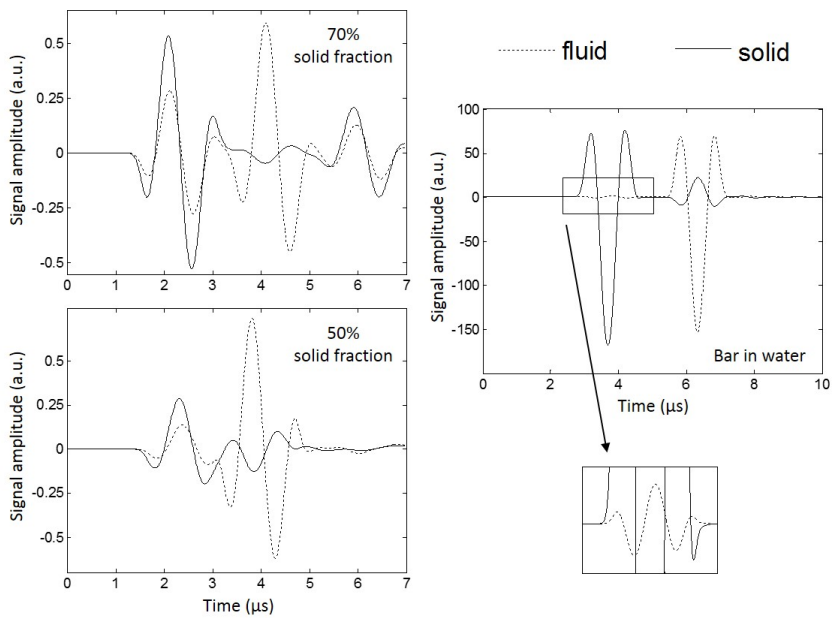

Figure 8: Left: particle velocity in the fluid and solid phases for a 70\% (top) or a $50 \%$ (bottom) solid fraction sample taken at a $4 \mathrm{~mm}$ propagation depth. Comparison with the particle velocity taken at a $8 \mathrm{~mm}$ propagation depth at each side of the interface of a solid bar in water (bottom right).

also studied the phase shifts between the motion of the fluid and solid phase in a very simple waveguide: a bar immersed in water. This particular case also exhibits two waves (Fig.8), with a guided wave in the bar re-radiating in water. Here too, the particle velocities are nearly in phase opposition for the slow wave $\left(177^{\circ}\right.$ shift). However the shift for the fast wave is around $131^{\circ}$ and so neither in nor out of phase. Though there is a discrepancy for the fast wave this result on a very simple case could be consistent with the fact that Biot's theory could be a particular, low frequency, case of a more general theory of the propagation of elastic waves in biphasic media involving guided waves.

\section{Conclusion}

The aim of this study was to compare quantitatively - in terms of velocities and attenuations - the results obtained from the simulation of elastic waves propagation in numerical anisotropic porous media with two theoretical approaches: Biot's model and a first-order multiple scattering model known as the Independent Scattering Approximation (ISA). The ISA was shown to provide good predictions of both velocity and attenuation coefficient when only one longitudinal wave occurred, and for low solid fractions (less than 10\%). It was however unsuccessful to predict the two compressional waves occurring from the propagation along the main orientation of the scatterers. On the other hand, homogenization theories such as Biot's or Wood's were found in good agreement with the numerical results, whether one or two waves were observed, but only for the velocities and not for the attenuations. This ${ }^{546}$ suggests that attenuation is probably better predicted based on multiple scattering theories. Yet at higher solid fractions, the $\mathrm{f}_{548}^{547}$ ISA will have to be replaced by higher-order approximations. ${ }_{549}$

\section{Acknowledgments}

Fabien Mézière is the recipient of a doctoral grant from the AXA Research Fund. We are also very grateful to Dr Patrick Rasolofosaon, from IFP-EN, for fruitful discussions on Biot's theory.

\section{Appendix A. Velocity of the fast wave}

Under the stiff frame assumption, Johnson [26] gives simple relations for fast, slow and transverse waves velocities. In particular the fast and transverse waves velocities can be written

$$
\begin{gathered}
V_{f a s t}=\sqrt{\frac{K_{d r y}+\frac{4}{3} N_{d r y}}{\Phi_{s} \rho_{s}+\Phi_{f} \rho_{f}\left(1-\frac{1}{\alpha}\right)}} \\
V^{T}=\sqrt{\frac{N_{d r y}}{\Phi_{s} \rho_{s}+\Phi_{f} \rho_{f}\left(1-\frac{1}{\alpha}\right)}}
\end{gathered}
$$

where $K_{d r y}$ and $N_{d r y}$ are the bulk and shear moduli of the dry sample (no filling fluid). When fluid is present, the stiff frame hypothesis is fulfilled as long as $K_{d r y} \gg K_{f}$ and $N_{d r y} \gg K_{f}$, with $K_{f}$ the bulk modulus of the fluid. In our case $K_{d r y}$ and $N_{d r y}$ were deduced from transverse $V_{d r y}^{T}$ and longitudinal $V_{d r y}^{L}$ velocities in the dry sample $\left(\rho_{f}=0\right)$ as follows:

$$
\begin{aligned}
& V_{d r y}^{L}=\sqrt{\frac{K_{d r y}+\frac{4}{3} N_{d r y}}{\Phi_{s} \rho_{s}}} \\
& V_{d r y}^{T}=\sqrt{\frac{N_{d r y}}{\Phi_{s} \rho_{s}}}
\end{aligned}
$$

As a consequence

$$
\begin{aligned}
& N_{d r y}=\Phi_{s} \rho_{s} V_{d r y}^{T^{2}} \\
& K_{d r y}=\Phi_{s} \rho_{s}\left(V_{d r y}^{L^{2}}-\frac{4}{3} V_{d r y}^{T}{ }^{2}\right)
\end{aligned}
$$

which means that Eq.A.1 can be advantageously simplified as in Sec. 2.4

$$
\begin{aligned}
V_{\text {fast }} & =\sqrt{\frac{\Phi_{s} \rho_{s} V_{d r y}^{L}{ }^{2}}{\Phi_{s} \rho_{s}+\Phi_{f} \rho_{f}\left(1-\frac{1}{\alpha}\right)}} \\
& =\frac{V_{d r y}^{L}}{\sqrt{1+\frac{\Phi_{f} \rho_{f}}{\Phi_{s} \rho_{s}}\left(1-\frac{1}{\alpha}\right)}}
\end{aligned}
$$

It is interesting to notice that according to this equation, the fast wave velocity does not depend on the transverse wave velocity, which was not so clear while looking at Eq.A.1.

\section{References}

[1] A. Hosokawa, T. Otani, Ultrasonic wave propagation in bovine cancellous bone., The Journal of the Acoustical Society of America 101 (1997) 55862 . 
[2] Z. E. A. Fellah, J. Y. Chapelon, S. Berger, W. Lauriks, C. Depollier, Ul-62 trasonic wave propagation in human cancellous bone: Application of Biot622 theory, The Journal of the Acoustical Society of America 116 (2004) 61.623

[3] K. Mizuno, Y. Nagatani, K. Yamashita, M. Matsukawa, Propagation624 of two longitudinal waves in a cancellous bone with the closed pore 625 boundary., The Journal of the Acoustical Society of America 130 (2011)626 EL122-7.

[4] T. Yamamoto, T. Otani, H. Hagino, H. Katagiri, T. Okano, I. Mano,62 R. Teshima, Measurement of human trabecular bone by novel ultrasonic 629 bone densitometry based on fast and slow waves., Osteoporosis Int 20630 (2009) 1215-24.

[5] A. B. Wood, A Textbook of Sound, Bell and Sons, 1955.

[6] M. A. Biot, Theory of Propagation of Elastic Waves in a Fluid-Saturated 633 Porous Solid. I. Low-Frequency Range, The Journal of the Acoustical634 Society of America 28 (1956) 168

[7] M. A. Biot, Theory of Propagation of Elastic Waves in a Fluid-Saturated 636 Porous Solid. II. Higher Frequency Range, The Journal of the Acoustical637 Society of America 28 (1956) 179.

[8] T. J. Plona, Observation of a second bulk compressional wave in a porous639 medium at ultrasonic frequencies, Applied Physics Letters 36 (1980) 259.

[9] R. Lakes, H. Yoon, J. Katz, Slow compressional wave propagation in wet human and bovine cortical bone, Science (1983) 513-515.

[10] Z. E. A. Fellah, N. Sebaa, M. Fellah, F. G. Mitri, E. Ogam, W. Lauriks, C. Depollier, Application of the biot model to ultrasound in bone: direct problem., IEEE transactions on ultrasonics, ferroelectrics, and frequency control 55 (2008) 1508-15.

[11] P. Laugier, G. Haiat, Bone Quantitative Ultrasound, Springer, 2011.

[12] A. Derode, V. Mamou, F. Padilla, F. Jenson, P. Laugier, Dynamic coherent backscattering in a heterogeneous absorbing medium: Application to human trabecular bone characterization, Applied Physics Letters 87 (2005) 114101.

[13] S. M. Rytov, Y. A. Kravtsov, V. I. Tatarskii, Principles of Statistical Radiophysics 4: Wave Propagation Through Random Media, Springer Verlag, Berlin Heidelberg, 1989.

[14] E. Akkermans, G. Montambaux, Mesoscopic Physics of Electrons and Photons, Cambridge University Press, 2007.

[15] J. Turner, Elastic wave propagation and scattering in heterogeneous, anisotropic media: Textured polycrystalline materials, Journal of the Acoustical Society of America 106 (1999) 541-552.

[16] M. Cowan, J. H. Page, P. Sheng, Ultrasonic wave transport in a system of disordered resonant scatterers: Propagating resonant modes and hybridization gaps, Physical Review B 84 (2011) 1-9.

[17] P. Sheng, Introduction to Wave Scattering, Localization and Mesoscopic Phenomena, Academic Press, New York, 1995.

[18] A. Hosokawa, T. Otani, Acoustic anisotropy in bovine cancellous bone., The Journal of the Acoustical Society of America 103 (1998) 2718-22.

[19] E. Bossy, F. Padilla, F. Peyrin, P. Laugier, Three-dimensional simulation of ultrasound propagation through trabecular bone structures measured by synchrotron microtomography., Physics in medicine and biology 50 (2005) 5545-56.

[20] G. Haïat, F. Padilla, F. Peyrin, P. Laugier, Fast wave ultrasonic propagation in trabecular bone: numerical study of the influence of porosity and structural anisotropy., The Journal of the Acoustical Society of America 123 (2008) 1694-705.

[21] Y. Nagatani, K. Mizuno, T. Saeki, M. Matsukawa, T. Sakaguchi, H. Hosoi, Numerical and experimental study on the wave attenuation in bone-FDTD simulation of ultrasound propagation in cancellous bone., Ultrasonics 48 (2008) 607-12.

[22] A. Hosokawa, Simulation of ultrasound propagation through bovine cancellous bone using elastic and Biot's finite-difference time-domain methods, The Journal of the Acoustical Society of America 118 (2005) 1782.

[23] F. Mézière, M. Muller, B. Dobigny, E. Bossy, A. Derode, Simulations of ultrasound propagation in random arrangements of elliptic scatterers: Occurrence of two longitudinal waves, The Journal of the Acoustical Society of America 133 (2013) 643-652.

[24] E. Bossy, M. Talmant, P. Laugier, Three-dimensional simulations of ultrasonic axial transmission velocity measurement on cortical bone models, The Journal of the Acoustical Society of America 115 (2004) 2314.

[25] A. Derode, V. Mamou, A. Tourin, Influence of correlations between scatterers on the attenuation of the coherent wave in a random medium, Physical Review E 74 (2006) 036606.
[26] D. L. Johnson, Equivalence between fourth sound in liquid $\mathrm{He}$ II at low temperatures and the Biot slow wave in consolidated porous media, Applied Physics Letters 37 (1980) 1065.

[27] K. A. Wear, Group velocity, phase velocity, and dispersion in human calcaneus in vivo, The Journal of the Acoustical Society of America 121 (2007) 2431.

[28] L. Forest, V. Gibiat, T. Woignier, Biot's theory of acoustic propagation in porous media applied to aerogels and alcogels, Journal of non-crystalline solids 225 (1998) 287-292.

[29] S. Chaffaï, F. Padilla, G. Berger, P. Laugier, In vitro measurement of the frequency-dependent attenuation in cancellous bone between 0.2 and 2 MHz., The Journal of the Acoustical Society of America 108 (2000) 1281-9.

[30] K. A. Wear, Ultrasonic Attenuation in Human Calcaneus from 0 . 2 to 1 . $7 \mathrm{MHz} 148$ (2001) 602-608.

[31] C. C. Anderson, K. R. Marutyan, M. R. Holland, K. A. Wear, J. G. Miller, Interference between wave modes may contribute to the apparent negative dispersion observed in cancellous bone., The Journal of the Acoustical Society of America 124 (2008) 1781-9. 